\title{
Estandarización de modelo de perfusión renal en ratones
}

\author{
Yángüez, Karen \\ Dpto. de Fisiología Humana, Fac. de medicina, Universidad de Panamá \\ Centro de Investigaciones Psicofarmacológicas, Panamá, Panamá \\ kyanguez@gmail.com \\ Díaz, Maricselis
}

Centro de Investigaciones Psicofarmacológicas, Fac. de medicina, Universidad de Panamá, Panamá

csifar.up@gmail.com

Morán, Juan

Centro de Investigaciones Psicofarmacológicas

Dpto. de Farmacología, Fac. de medicina, Universidad de Panamá,

Panamá, coljamp@gmail.com

\section{Mero, Aldahir}

Centro de Investigaciones Psicofarmacológicas

Dpto. de Farmacología, Fac. de medicina, Universidad de Panamá, Panamá

csifar.up@gmail.com

\section{Chérigo, Katherine}

Centro de Investigaciones Psicofarmacológicas

Fac. de medicina, Universidad de Panamá, Panamá

csifar.up@gmail.com

\section{Aizprúa, Betsy}

Centro de Investigaciones Psicofarmacológicas

Fac. de medicina, Universidad de Panamá, Panamá

csifar.up@gmail.com

\section{Navarro, Alireya}

Centro de Investigaciones Psicofarmacológicas

Fac. de medicina, Universidad de Panamá, Panamá

csifar.up@gmail.com 


\section{Francis, Nayaris}

Centro de Investigaciones Psicofarmacológicas

Fac. de medicina, Universidad de Panamá, Panamá

csifar.up@gmail.com

\section{Moráles, Abdy}

Centro de Investigaciones Psicofarmacológicas

Dpto. de Farmacología, Fac. de medicina, Universidad de Panamá, Panamá

csifar.up@gmail.com

\section{Jaén, Delia}

Dpto. de Fisiología Humana, Fac. de medicina, Universidad de Panamá, Panamá deliajaendegarrido@gmail.com

\section{Guerrero De León, Estela}

Sistema Nacional de Investigación Centro de Investigaciones Psicofarmacológicas

Dpto. de Farmacología, Fac. de Medicina, Universidad de Panamá, Panamá guerrerodleon@gmail.com

\section{ABSTRACT}

Ex vivo kidney technique allows the intrinsic renal response to be assessed, allowing the experimental variables to be controlled by the investigator. In the CIPFAR laboratories we worked to fine-tune the isolated and perfused kidney model, for which we used C57BL/6 and CD-1 male mice. We performed abdominal laparatomy, located and ligated the aortic section, then made an incision in the left renal artery and introduced a cannula attached to a polyethylene microtube. Through the cannula, the isolated kidneys are connected to a perfusion system and exposed to the contracting agents $\mathrm{KCl}(80 \mathrm{mM})$ and phenylephrine (FE 10-5 M). We also obtained concentration-response curves against acetylcholine (ACh 10-810-4M). Our results reveal basal pressure values of $36.8 \pm 4.8 \mathrm{mmHg}$ and $30.7 \pm 14.2 \mathrm{mmHg}$ for CD-1 and C57BL/6 strains, respectively. Depolarization with $\mathrm{KCl}$ developed a maximum contracting effect of $25.7 \pm 16.0 \mathrm{mmHg}$ for $\mathrm{CD}-1$ and $63.8 \pm 34.8 \mathrm{mmHg}$ in C57BL/6 mice. The adrenergic agent FE produced maximal contractions of $53.0 \pm 29.6 \mathrm{mmHg}$ in $\mathrm{CD}-1$ and $66.1 \pm 4.7 \mathrm{mmHg}$ in $\mathrm{C} 57 \mathrm{BL} / 6$. The maximum ACh-induced vasodilator effect was $35.7 \% \pm 3.5$ and $46.9 \% \pm 18.7$ in CD-1 and C57BL/6, respectively.

In light of this result, both exposure time, excessive handling, inadvertent bubble perfusion and clot formation may be factors that decrease renal endothelial viability. In any case, basal perfusion data as well as reactivity to contracting agents are evidence of tissue viability in both groups of animals.

Keywords: Isolated kidney, Vasodilator, Vasoconstrictor, renal function, perfusion. 


\section{RESUMEN}

La técnica de riñón ex vivo permite valorar la respuesta renal intrínseca permitiendo que las variables experimentales puedan ser controladas por el investigador. En los laboratorios del CIPFAR trabajamos para poner a punto el modelo de riñón aislado y perfundido, para lo cual empleamos ratones C57BL/6 y CD-1 machos. Realizamos laparatomía abdominal, localizamos y ligamos la sección aórtica, seguidamente, realizamos una incisión en la arteria renal izquierda e introdujimos una cánula acoplada a un microtubo de polietileno. A través de la cánula, los riñones aislados son conectados a un sistema de perfusión y expuestos a los contracturantes $\mathrm{KCl}(80 \mathrm{mM})$ y fenilefrina (FE 10-5 M). También obtuvimos curvas concentración respuesta frente a acetilcolina (ACh 10-8-10-4M). Nuestros resultados revelan valores de presión basal de $36.8 \pm 4.8 \mathrm{mmHg}$ y $30.7 \pm 14.2 \mathrm{mmHg}$ para las cepas CD-1 y C57BL/6, respectivamente. La despolarización con $\mathrm{KCl}$ desarrolló un efecto contracturante máximo de $25.7 \pm 16.0 \mathrm{mmHg}$ para CD-1 y $63.8 \pm 34.8 \mathrm{mmHg}$ en ratones $\mathrm{C} 57 \mathrm{BL} / 6$. El agente adrenérgico $\mathrm{FE}$, produjo contracciones máximas de $53.0 \pm 29.6 \mathrm{mmHg}$ en $\mathrm{CD}-1$ y $66.1 \pm 4.7$ $\mathrm{mmHg}$ en $\mathrm{C} 57 \mathrm{BL} / 6$. El efecto vasodilatador máximo inducido por ACh fue de $35.7 \% \pm 3.5 \mathrm{y}$ $46.9 \% \pm 18.7$ en $\mathrm{CD}-1$ y $\mathrm{C} 57 \mathrm{BL} / 6$, respectivamente.

A la luz de este resultado, tanto el tiempo de exposición, la manipulación excesiva, la perfusión inadvertida de burbujas y la formación de coágulos, pueden ser factores que disminuyan la viabilidad del endotelio renal. En cualquier caso, los datos de perfusión basal, así como la reactividad frente a agentes contracturantes son pruebas de la viabilidad del tejido en ambos grupos de animales.

Palabras claves: Riñón aislado, Vasodilatador, Vasoconstrictor, Función Renal, Perfusión.

\section{INTRODUCCIÓN}

Las patologías renales en los últimos años han tenido una relevancia epidemiológica creciente, por elloactualmente son identificadas como un problema de salud pública [1]. En Panamá instituciones como: Caja de Seguro Social e Instituto Conmemorativo Gorgas de Estudios de la Salud, tienen contemplado seguir realizando estudios dirigidos a cuantificar la prevalencia e incidencia de las patologías renales en la población panameña. Es oportuno desarrollar estandarizaciones Ex vivo e In vitro de modelos experimentales en nefrología, los cuales se convertirán en aportes significativos en investigaciones enfocadas en el estudio de la fisiopatología del riñón. 
El riñón juega un papel importante en la eliminación de toxinas y productos metabólicos de la sangre, así como también sobre la regulación de los metabolitos en el organismo. Si bien es cierto que la realización de investigación sobre la función renal es esencial para comprender el manejo y eliminación de metabolitos, suele ser complicada al utilizar animales completos debido a la influencia metabólica de los tejidos extrarrenales [2]; [3]. En este sentido, la técnica del riñón aislado y perfundidoofrece la ventaja de trabajar sobre el riñón permitiendo al investigador modificar las variables experimentales de manera controlada.

La técnica de riñón aislado y perfundido permite valorar la función renal a través de la respuesta intrarrenal (autorregulación) del riñón, sin la intervención de la actividad neurohormonal sistémica o respuesta extrarrenal [4].

En general, esta técnica permite la evaluación continua de la tasa de flujo renal, la resistencia vascular,la liberación de hormonas, el análisis de gases en sangre, el análisis de orina y la aplicación de fármacos. Después del procedimiento, el riñón puede ser procesado para biología molecular, estudios metabolómicos, análisis bioquímico o microscopía [5].

En la literatura se reportan múltiples utilidades de la técnica de estandarización de modelo de perfusiónrenal en ratones para las investigaciones farmacológicas y fisiológicas de enfermedades crónicas [6][7] [8] [4].

Esta técnica ya ha sido empleada en ratones C57BLI/6 [6], sin embargo, ajustándonos a las cepas disponibles en los bioterios de la Universidad de Panamá y del INDICASAT-AIP, nos proponemos estandarizar la técnica de riñón aislado y perfundido en ratones CD-1 y C57BL/6, los cuales son a los que tiene acceso la mayoría de la comunidad científica en nuestro país, para las investigaciones fisiológicas y farmacológicas ex vivo.

\section{MÉTODO}

\section{A. Modelo de estudio}

Se emplearon ratones machos, entre 25 y $30 \mathrm{~g}$, de las cepas CD-1 del Bioterio de la Universidad de Panamá y C57BL/6 provenientes del Bioterio de INDICASATAIP. Los animales fueron aclimatados, con acceso libre a comida y agua, según los criterios establecidos en la Guía para el cuidado y uso de animales de laboratorio publicado por el Nacional Health Institute, 2011. El estudio fue aprobado por el Comité de Bioética de la Universidad de Panamá (N CBUP/236/2019).

\section{B. Procedimiento experimental}

Los animales fueron anestesiados con pentobarbital sódico $(50 \mathrm{mg} / \mathrm{kg})$ y anticoagulados con heparina sódica $(500 \mathrm{U} / \mathrm{kg})$. 
Se procedió a realizar una laparotomía abdominal para localizar y ligar la sección aórtica directamente debajo de la arteria renal derecha y encima de la izquierda, seguidamente, se realizó una incisión en laarteria renal izquierda a través de la cual se introdujo una cánula acoplada a un microtubo depolietileno $(1.19 \mathrm{~mm} \times 1.70 \mathrm{~mm})$. Seguidamente, se preparó el paquete, riñón-cánula, para llevarlo al sistema de perfusión aislada (Fig. 1).

A través de la cánula, el riñón fue conectado a una llave de tres vías permitiendo de forma simultánea la perfusión del gas carbógeno compuesto por una mezcla de $\mathrm{O}_{2}-\mathrm{CO}_{2}$ (19:1) y la conexión al sistema de registro en donde la presión de perfusión es obtenida mediante un transductor de presión para ser registrada de manera continua. Posteriormente, se inició la perfusión con solución Krebs a 37으 burbujeada continuamente con una mezcla de carbógeno, a una velocidad de $1 \mathrm{~mL} / \mathrm{min}$.

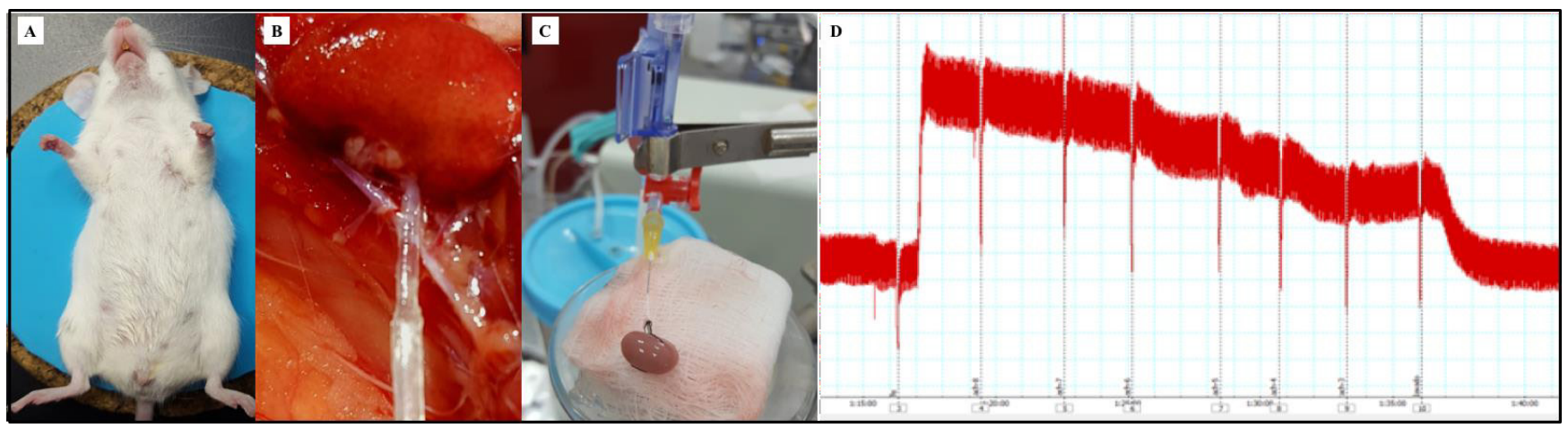

Figura 1. Realización de laparotomía abdominal bajo anestesia (A), localización y canulación del riñón izquierdo (B), colocación de la preparación cánula-riñón en el sistema de perfusión (C), Registro de la curva concentración- respuesta a la Acetilcolina (D).

Se dejó estabilizar la preparación durante 15 minutos para luego determinar la presión de perfusión basal. Los riñones fueron expuestos a una solución de $\mathrm{KCl}$ a $80 \mathrm{mM}$ seguido de un lavado con solución Krebs a 37ํㅡ y después de recuperarse y lograr tener una perfusión basal, se procedió a la obtención de la curva concentración-respuesta a acetilcolina (ACh $10^{-8}-10^{-4} \mathrm{M}$ ) en presencia de una contracción con Fenilefrina $1 \times 10^{-5} \mathrm{M}$ (Fig. 1D).

\section{RESULTADOS}

El modelo empleado para medir los cambios de perfusión renal utilizando riñones aislados permitió mantener el riñón de ratón ex vivo perfundido y funcional al menos 1 hora para la obtención de datos. Los resultados revelan que los valores de presión basal fueron de $36.8 \pm 4.8$ y $30.7 \pm 14.2 \mathrm{mmHg}$ para las cepas CD-1 y C57BL/6, respectivamente, los cuales se encuentran dentro de rango normal establecido que oscila entre $20 \mathrm{mmH}$ y $120 \mathrm{mmHg}$ (Fig. $2 \mathrm{~A}$ ). 
En los riñones de ratones CD-1, la despolarización sobre el músculo liso vascular efectuada a través d la perfusión con $\mathrm{KCl}$, desarrolló un efecto contracturante máximo de $25.7 \pm 16.0 \mathrm{mmHg}$, mientras que en ratones $\mathrm{C} 57 \mathrm{BL} / 6$ el mismo agente, alcanzó una presión máxima de $63.8 \pm 34.8 \mathrm{mmHg}$ (Fig. $2 \mathrm{~B}$ ).

El agente adrenérgico fenilefrina, produjo contracciones máximas de $53.0 \pm 29.6$ y 66.1 $\pm 4.7 \mathrm{mmHg}$ en ratones CD-1 y C57BL/6, respectivamente (Fig. $3 \mathrm{C}$ ). Por otro lado, el efecto vasodilatador máximo obtenido con ACh fue del $35.7 \% \pm 3.5$ y $46.9 \% \pm 18.7$ en ratones CD-1 y C57BL/6, respectivamente (Fig. 3 D).

Al comparar los valores de presión basal observamos que estos fueron similares para ambas cepas, mientras que la actividad contracturante mediada por despolarización ( $\mathrm{KCl}$ ) y por estimulación de receptores adrenérgicos alfa-1 (FE), fue mayor en riñones perfundidos aislados de ratones $\mathrm{C} 57 \mathrm{BL} / 6$. En animales jóvenes y sin condiciones patológicas el efecto máximo reportado para ACh es entre el 60 y 70\%, sin embargo, en ninguno de los grupos alcanzamos valores de relajación que reflejen integridad total del tejido endotelial. Es importante tomar en cuenta que la ACh ha sido ampliamente utilizada como vasodilatador endotelio-dependiente, lo que implica reconocer que su efecto difiere dependiendo del lecho vascular, teniendo en cuenta que la respuesta vasodilatadora se produce cuando el endotelio está intacto.

A la luz de este resultado, consideramos que el tiempo de exposición, la manipulación excesiva, la perfusión inadvertida de burbujas y la formación de coágulos, pueden ser factores que afecten la viabilidad del endotelio renal, reduciendo la eficacia vasodilatadora de la acetilcolina; la cual se espera que sea superior al $70 \%$ en un sistema con endotelio funcional.

De la utilidad de esta técnica podemos afirmar que, durante el tiempo de la perfusión, se puede manipular el riñón, evaluar la función renal y administrar diversos productos farmacéuticos. Después del procedimiento, el riñón puede ser procesado para biología molecular, estudios metabolómicos, análisis bioquímico o microscopía. 
A

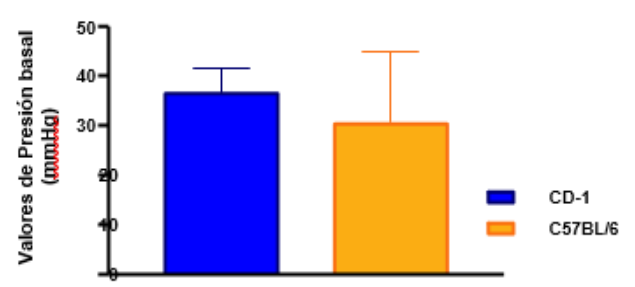

C

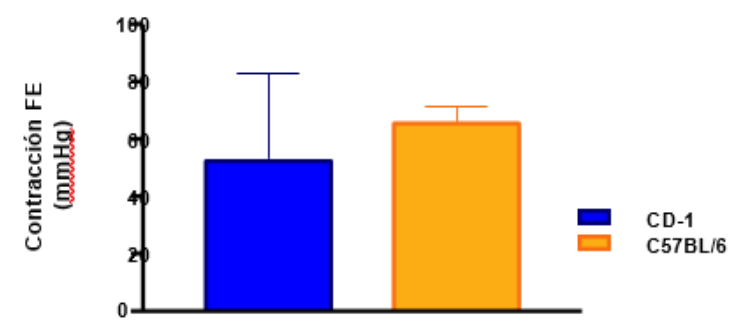

B

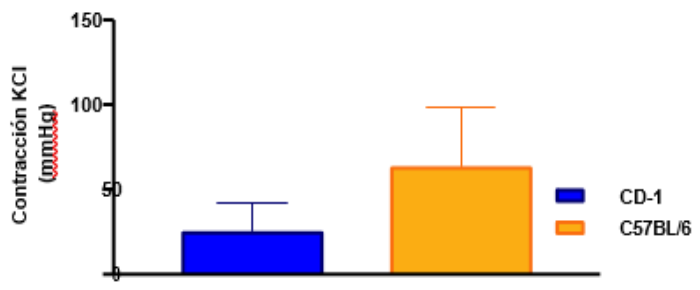

D

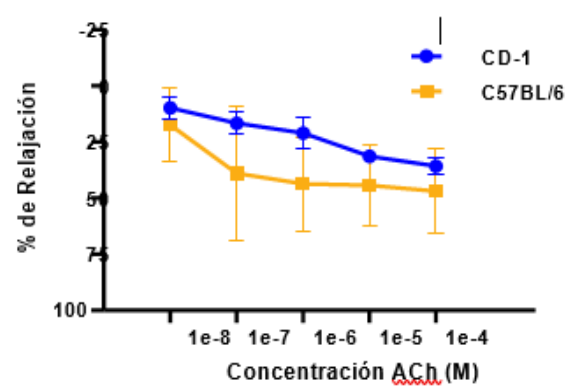

Figura 2: Medida de la funcionalidad renal ex vivo en riñones de ratones CD-1 y C57BL/6. (A) Presión basal de perfusión $(\mathrm{mmHg})$. (B) Contracción en presencia de $\mathrm{KCl}$ a una concentración de $80 \mathrm{mM}$. (C) Contracción en presencia de fenilefrina $1 \times 10^{-5} \mathrm{M}$. (D) Porcentaje de relajación en reportado durante la curvaconcentración-respuesta a Acetilcolina en presencia de Fenilefrina $1 \times 10^{-5} \mathrm{M}$.

\section{CONCLUSIONES}

El riñón aislado perfundido de ratón es una herramienta para estudiar la función renal en un entorno controlado ex vivo. La técnica presentada se puede utilizar para la realización de estudios sobre la fisiología renal, y más recientemente como herramienta para dilucidar el papel de las intervenciones farmacológicas en modelos de enfermedad renal.

Aunque logramos presiones de perfusión basal adecuadas y que el tejido respondió a los agentes contracturantes, la falta de eficacia vasodilatadora frente a la acetilcolina hace que debamos plantearnos algunas derivaciones protocolares para alcanzar efectos vasorelajantes óptimos. Así, para obtener una viabilidad endotelial máxima, las intervenciones quirúrgicas deben realizarse en no más de 30 minutos. Por otra parte, se debe asegurar el correcto funcionamiento del sistema de perfusión durante todo el experimento, controlando y verificando la temperatura del perfundido, la velocidad de perfusión, evitar que se formen burbujas en el circuito de perfusión. Los datos representativos proporcionados nos permiten concluir que tanto la cepa CD-1 como la C57BL/6 podrán ser utilizadas para llevar a cabo este modelo. 


\section{RECONOCIMIENTO}

Este trabajo fue realizado bajo el Proyecto de investigación IOMS19-002 con fondos otorgados por la SENACYT, con el apoyo del Sistema Nacional de Investigación (Investigador Nacional/ Estela Guerrero).

\section{REFERENCIAS}

[1] C. Juan, J. C. Flores, M. Alvo, H. Borja, J. Vega, and C. Zúñiga, "Sociedad Chilena de Nefrología Enfermedad renal crónica : Clasificación, identificación, manejo y complicaciones Clinical guidelines on identification , management and complications of chronic kidney disease," 2009.

[2] M. P. Hoenig and M. L. Zeidel, "Renal Physiology the Nephron," vol. 9, 2014.

[3] H. Chang, B. Choong, A. Phillips, and K. M. Loomes, "The Isolated Perfused Rat Kidney : A Technical Update," vol. 62, no. June 2012, pp. 19-23, 2013.

[4] L. Trumper and L. A. Monasterolo, "Modelos experimentales en nefrología. modelo de riñón aislado y perfundido," pp. 115-118, 2015.

[5] J. Czogalla, "A protocol for rat kidney normothermic machine perfusion and subsequent transplantation," no. May, pp. 1-7, 2020.

[6] S. Jain, R. Plenter, T. Nydam, and A. Jani, "Injury Pathways That Lead to AKI in a Mouse Kidney Transplant Model," vol. 104, no. 9, pp. 1832-1841, 2020.

[7] H. Nagasu et al., "Endothelial dysfunction promotes the transition from compensatory renal hypertrophy to kidney injury after unilateral nephrectomy in mice," pp. 1402-1408, 2021.

[8] J. D. Imig, M. D. Breyer, R. M. Breyer, D. John, M. D. Breyer, and M. Richard, "Contribution of prostaglandin EP 2 receptors to renal microvascular reactivity in mice," pp. 415-422, 2002.

\section{AUTORIZACIÓN Y LICENCIA CC}

Los autores autorizan a APANAC XVIII a publicar el artículo en las actas de la conferencia en Acceso Abierto (Open Access) en diversos formatos digitales (PDF, HTML, EPUB) e integrarlos en diversas plataformas online como repositorios y bases de datos bajo la licencia CC: Attribution-NonCommercial-ShareAlike 4.0 International (CC BY-NC-SA 4.0) https://creativecommons.org/licenses/by-ncsa/4.0/.

Ni APANAC XVIII ni los editores son responsables ni del contenido ni de las implicaciones de loexpresado en el artículo. 